\title{
THE SPATIALLY HOMOGENEOUS RELATIVISTIC BOLTZMANN EQUATION WITH A HARD POTENTIAL
}

\author{
HO LEE AND ALAN D. RENDALL
}

\begin{abstract}
In this paper, we study spatially homogeneous solutions of the Boltzmann equation in special relativity and in Robertson-Walker spacetimes. We obtain an analogue of the Povzner inequality in the relativistic case and use it to prove global existence theorems. We show that global solutions exist for a certain class of collision cross sections of the hard potential type in Minkowski space and in spatially flat Robertson-Walker spacetimes.
\end{abstract}

\section{INTRODUCTION}

Under the assumption of homogeneity the non-relativistic Boltzmann equation has been extensively studied for many years. Homogeneity means that the unknown in the equation, which in general depends on time, spatial variables and velocity variables, is restricted to depend only on time and velocity variables. Since Carleman gave the first proof of global existence [5] in the 1930s, many mathematicians have obtained results on the homogeneous non-relativistic Boltzmann equation, and by now a rather complete mathematical theory is available. In the present paper we are interested in generalizations of this to special and general relativity. Compared to the non-relativistic case, the relativistic equations have not been studied much. Noutchegueme and his colleagues obtained results on the homogeneous Boltzmann equation in 18, 19, 20, 21 in several different relativistic situations, but the scattering kernels they used are not physically well-motivated. The purpose of this paper is to obtain analogues of their results in more physically relevant cases.

The scattering kernel is a quantity that determines the nature of collisions between particles, and in the non-relativistic case several different types of scattering kernel have been found to be of interest. For instance, the inverse power law gives the best-known types of scattering kernel, and they are further classified into hard and soft potential cases. In the relativistic setting it is not so clear which types of scattering kernel should be of interest, but a classification of (special) relativistic hard and soft potentials has been proposed in [9, 28, by applying arguments similar to those used in the non-relativistic case. As in the non-relativistic case, the scattering kernels depend only on the relative momentum and scattering angle of two colliding particles. Consider a collision of two particles, and let $p^{\alpha}$ and $q^{\alpha}$ be their momenta before the collision, and $p^{\prime \alpha}$ and $q^{\prime \alpha}$ the momenta after the collision. Then the scattering kernel is given by a function of $p^{\alpha}-q^{\alpha}$ and $p^{\alpha}-q^{\prime \alpha}$. To be precise, the scattering kernel depends only on the following two quantities:

$$
\left(p_{\alpha}-q_{\alpha}\right)\left(p^{\alpha}-q^{\alpha}\right) \text { and }\left(p_{\alpha}-q_{\alpha}\right)\left(p^{\prime \alpha}-q^{\prime \alpha}\right)
$$

where the indices are lowered by the Minkowski metric. The above two quantities are related to the relative momentum and the scattering angle respectively, and the relativistic hard and soft potentials are defined in terms of the above quantities. 
Their precise definitions will be given in Sections 1.1 and 1.2. On the other hand, the general relativistic Boltzmann equation does not seem to have been studied enough concerning specific types of scattering kernel. For instance, in 3, 4, the authors introduced a quantity $S$ which is a function of $x, p, q, p^{\prime}$, and $q^{\prime}$. The quantity $S$ was used to play the role of the kernel of the collision operator of the Boltzmann equation, and strong assumptions on $S$ were made to obtain local well-posedness of the Einstein-Boltzmann system. However, instead of introducing the abstract quantity, we may use the principle of general covariance to deal with the collision operator. If we write down the collision operator with an arbitrary Lorentzian metric replacing the Minkowski metric, then the special relativistic collision operator is extended to general relativity in a natural way. This argument is consistent with the following fact. There exists an alternative way to write the general relativistic Boltzmann equation. One can introduce an orthonormal frame $e_{\mu}^{\alpha}$ on spacetime and parametrize the mass shell as $p^{\alpha}=e_{\mu}^{\alpha} v^{\mu}$. Then, the collision operator no longer contains any explicit dependence on the metric and reduces to the special relativistic collision operator. We refer to [16] for a more detailed discussion of this approach.

In this paper, we follow the procedure just mentioned. The general relativistic collision operator has the same form as in the special relativistic case, but a certain type of Lorentzian metric will replace the Minkowski metric. The definitions of hard and soft potentials will be understood by using the principle of general covariance, and eventually we will show that the Boltzmann equation has a global solution in the case of a certain type of hard potential in spatially flat Robertson-Walker spacetimes. We refer to 25, 31 for basic information about general relativity and to [1, 8, 10, 27] and Chapter $\mathrm{X}$ of [7] for relativistic kinetic theory. For classical theories of the non-relativistic Boltzmann equation we refer to [6, 12] and their references.

1.1. Remarks on the relativistic Boltzmann equation. Since the Boltzmann equation is an equation describing the dynamics of collisional matter, it is necessary to understand the collision processes between particles in order to investigate the equation. The main difference between the non-relativistic and relativistic collision processes is that energy and momentum conservation in the non-relativistic case are replaced by an energy-momentum conservation, and this causes a difficulty in parametrizing post-collisional momenta in the relativistic case. Let $p^{\alpha}$ and $q^{\alpha}$ be two four-vectors describing momenta of two colliding particles in a relativistic situation, and suppose that the two particles produce momenta $p^{\alpha}$ and $q^{\prime \alpha}$ after the collision. Then, energy-momentum conservation is written as

$$
p^{\alpha}+q^{\alpha}=p^{\alpha}+q^{\prime \alpha} .
$$

Moreover, if all the particles are assumed to have the same mass, then the mass shell conditions

$$
p_{\alpha} p^{\alpha}=q_{\alpha} q^{\alpha}=p_{\alpha}^{\prime} p^{\prime \alpha}=q_{\alpha}^{\prime} q^{\prime \alpha}=-1
$$

are additionally imposed. Since the Greek index $\alpha$ runs from 0 to 3 , we have six constraints and two free parameters for the post-collisional momenta, and for the free parameters we use $\omega \in \mathbb{S}^{2}$ as usual. Consequently, the post-collisional momenta $p^{\alpha}$ and $q^{\alpha}$ can be parametrized in terms of the pre-collisional momenta $p^{\alpha}$ and $q^{\alpha}$ with an additional parameter $\omega \in \mathbb{S}^{2}$. There are several different ways known to parametrize post-collisional momenta in the special relativistic case, see for instance 
[13, 29. However, the parametrization suggested in [16] will be used in this paper because it can be applied effectively to the general relativistic case. Suppose that $p^{\alpha}$ and $q^{\alpha}$ are given, and define

$$
n^{\alpha}:=p^{\alpha}+q^{\alpha} \quad \text { and } \quad t^{\alpha}:=\left(n_{i} \omega^{i},-n_{0} \omega\right)
$$

for $\omega \in \mathbb{S}^{2}$. Note that $t^{\alpha}$ is a general form of vectors orthogonal to $n^{\alpha}$, i.e. $n_{\alpha} t^{\alpha}=0$ for any $\omega \in \mathbb{S}^{2}$. The post-collisional momenta are represented as

$$
p^{\alpha}=\frac{p^{\alpha}+q^{\alpha}}{2}+\frac{g}{2} \frac{t^{\alpha}}{\sqrt{t_{\beta} t^{\beta}}} \quad \text { and } \quad q^{\prime \alpha}=\frac{p^{\alpha}+q^{\alpha}}{2}-\frac{g}{2} \frac{t^{\alpha}}{\sqrt{t_{\beta} t^{\beta}}} .
$$

It can easily be checked that they satisfy the mass shell condition and energymomentum conservation. The scalar quantity $g$ is called the relative momentum and is defined by (1.3) below.

In this paper we are mainly interested in the Robertson-Walker spacetimes but first we consider the related case of Minkowski space. The spatially homogeneous Boltzmann equation in Minkowski space is written as follows:

$$
\partial_{t} f=Q(f, f):=\int_{\mathbb{R}^{3}} \int_{\mathbb{S}^{2}} v_{\phi} \sigma(g, \theta)\left(f^{\prime} f_{*}^{\prime}-f f_{*}\right) \sqrt{|\eta|} d \omega d q, \quad v_{\phi}:=\frac{g \sqrt{s}}{p^{0} q^{0}},
$$

where $f^{\prime}=f\left(t, p^{\prime}\right), f_{*}^{\prime}=f\left(t, q^{\prime}\right), f=f(t, p)$, and $f_{*}=f(t, q)$. Here, $f$ is called the distribution function, $Q$ the collision operator, $v_{\phi}$ the Møller velocity, $\sigma$ the scattering kernel, $\theta$ the scattering angle, and $\eta$ the determinant of the Minkowski metric. The first component $p^{0}$ can be solved for in terms of the other components $p^{i}$ due to the mass shell condition as

$$
p^{0}=\sqrt{1+|p|^{2}}
$$

where $p=\left(p^{1}, p^{2}, p^{3}\right)$ denotes the spatial projection of $p^{\alpha}$, and the scalar quantities $s$ and $g$ are given by

$$
s:=-\left(p_{\alpha}+q_{\alpha}\right)\left(p^{\alpha}+q^{\alpha}\right), \quad g:=\sqrt{\left(p_{\alpha}-q_{\alpha}\right)\left(p^{\alpha}-q^{\alpha}\right)} .
$$

They are called the total energy and the relative momentum respectively. Note that they are conserved quantities in the collision process, i.e.

$$
s=-\left(p_{\alpha}^{\prime}+q_{\alpha}^{\prime}\right)\left(p^{\alpha}+q^{\prime \alpha}\right), \quad g=\sqrt{\left(p_{\alpha}^{\prime}-q_{\alpha}^{\prime}\right)\left(p^{\prime \alpha}-q^{\prime \alpha}\right)} .
$$

The scattering angle $\theta$ is finally defined as the angle between $p^{\alpha}-q^{\alpha}$ and $p^{\prime \alpha}-q^{\prime \alpha}$. It can be expressed in terms of Lorentz invariant quantities as follows:

$$
\cos \theta:=\frac{\left(p_{\alpha}-q_{\alpha}\right)\left(p^{\alpha}-q^{\alpha}\right)}{g^{2}}=1-2 \frac{\left(p_{\alpha}-p_{\alpha}^{\prime}\right)\left(p^{\alpha}-p^{\prime \alpha}\right)}{s-4},
$$

where $s$ and $\left(p_{\alpha}-p_{\alpha}^{\prime}\right)\left(p^{\alpha}-p^{\alpha}\right)$ are two of the Mandelstam variables. Note that the scattering angle does not depend on how the post-collisional momenta are parametrized.

In the second part of the paper we will consider the case of the spatially flat Robertson-Walker spacetimes. For a given scale factor $R(t)$, the Boltzmann equation is written as

$$
\partial_{t} f-2 \frac{\dot{R}}{R} \sum_{i=1}^{3} p^{i} \partial_{p^{i}} f=Q(f, f),
$$

where $\dot{R}$ denotes the time derivative of $R$. The collision operator $Q$ is the same as in (1.2), but all the quantities in it such as (1.3) and (1.4) are calculated through 
the Robertson-Walker metric. For instance, $\sqrt{|\eta|}$ is replaced by $R^{3}(t)$, and indices are lowered by the Robertson-Walker metric, so we have

$$
p^{0}=\sqrt{1+R^{2}(t)|p|^{2}}
$$

by the mass shell condition. However, in this paper we will take a different approach. It is well-known that in the Robertson-Walker case the Vlasov equation can be explicitly solved for any given initial data. If the distribution function is expressed in terms of covariant variables, then it is independent of time, hence we have $f\left(t, p_{i}\right)=f_{0}\left(p_{i}\right)$ in the Vlasov case. Similarly, we can use covariant variables for the Boltzmann equation. Let us consider again the collision process by using covariant variables. To make the difference between contravariant and covariant variables clear, we will use $v_{\alpha}$ for covariant variables, i.e.

$$
v_{\alpha}:=p_{\alpha} \quad \text { and } \quad v=\left(v_{1}, v_{2}, v_{3}\right) .
$$

Then, the energy-momentum conservation is written as

$$
v_{\alpha}+u_{\alpha}=v_{\alpha}^{\prime}+u_{\alpha}^{\prime}
$$

and the post-collisional momenta $v_{\alpha}^{\prime}$ and $u_{\alpha}^{\prime}$ are given by

$$
v_{\alpha}^{\prime}=\frac{v_{\alpha}+u_{\alpha}}{2}+\frac{g}{2} \frac{t_{\alpha}}{\sqrt{t_{\beta} t^{\beta}}} \quad \text { and } \quad u_{\alpha}^{\prime}=\frac{v_{\alpha}+u_{\alpha}}{2}-\frac{g}{2} \frac{t_{\alpha}}{\sqrt{t_{\beta} t^{\beta}}},
$$

where $g$ and $t^{\alpha}$ are basically the same as in the contravariant case, but they should be understood as quantities which are constructed from $v_{\alpha}$ and $u_{\alpha}$. Consequently, if the distribution function is regarded as a function of $t$ and $v$, instead of $p$, then the Boltzmann equation is written as

$$
\partial_{t} f=Q(f, f)=R^{-3} \int_{\mathbb{R}^{3}} \int_{\mathbb{S}^{2}} v_{\phi} \sigma(g, \theta)\left(f^{\prime} f_{*}^{\prime}-f f_{*}\right) d \omega d u, \quad v_{\phi}=\frac{g \sqrt{s}}{v_{0} u_{0}},
$$

where $f^{\prime}=f\left(t, v^{\prime}\right), f_{*}^{\prime}=f\left(t, u^{\prime}\right), f=f(t, v)$, and $f_{*}=f(t, u)$. The first component $v_{0}$ is solved for in terms of the other components using the mass shell condition as

$$
v_{0}=-\sqrt{1+R^{-2}(t)|v|^{2}}
$$

and the scalar quantities $s$ and $g$ and the scattering angle $\theta$ are the same as (1.3) and (1.4) respectively. The factor $R^{-3}$ comes from the relation

$$
R^{3}(t) d q=R^{-3}(t) d u .
$$

In the present paper the Minkowski case and the Robertson-Walker case will be studied separately. In the former case the Boltzmann equation will refer to (1.2), while in the latter case the equation will refer to (1.5). Comparing the two forms (1.2) and (1.5), we might expect that results in the Minkowski case could be easily extended to the Robertson-Walker case, and this will be done in Section 3 .

1.2. Assumptions of the paper. The scattering kernel $\sigma$ is a function of the relative momentum $g$ and the scattering angle $\theta$. According to the way that the scattering kernel depends on its variables, it is classified into hard and soft potentials. The classification of hard and soft potentials in the relativistic case was originally introduced by Dudyński and Ekiel-Jeżewska 9] and recently reformulated 
by Strain 28 as follows: for soft potentials we assume that there exist $\gamma>-2$ and $0<b<\min \{4, \gamma+4\}$ satisfying

$$
\begin{aligned}
\left(\frac{g}{\sqrt{s}}\right) g^{-b} \sigma_{0}(\omega) & \lesssim \sigma(g, \omega) \lesssim g^{-b} \sigma_{0}(\omega), \\
\sigma_{0}(\omega) & \lesssim \sin ^{\gamma} \theta
\end{aligned}
$$

while for hard potentials we assume that there exist $\gamma>-2,0 \leq a \leq \gamma+2$, and $0 \leq b<\min \{4, \gamma+4\}$ satisfying

$$
\begin{gathered}
\left(\frac{g}{\sqrt{s}}\right) g^{a} \sigma_{0}(\omega) \lesssim \sigma(g, \omega) \lesssim\left(g^{a}+g^{-b}\right) \sigma_{0}(\omega), \\
\sigma_{0}(\omega) \lesssim \sin ^{\gamma} \theta .
\end{gathered}
$$

Here for any two quantities $A, B$ the relation $A \lesssim B$ mens that there exists a constant $C$ such that $A \leq C B$.

The scattering kernel. In this paper we assume that the scattering kernel has the form

$$
\sigma(g, \omega)=g^{a} \sin ^{\gamma} \theta, \quad-2<\gamma \leq-1, \quad 0 \leq a \leq \gamma+2 .
$$

Since $\left(\frac{g}{\sqrt{s}}\right)$ is a bounded quantity (note that $s=4+g^{2}$ ), a scattering kernel of this form falls into the hard potential case.

Throughout the paper we use weighted $L^{1}$ spaces in momentum variables. Let $L^{1}\left(\mathbb{R}^{3}\right)$ be the usual Lebesgue space of integrable functions on $\mathbb{R}^{3} \cdot L_{r}^{1}\left(\mathbb{R}^{3}\right)$ denotes the weighted $L^{1}$ space with norm

$$
\|f\|_{1, r}:=\int_{\mathbb{R}^{3}} f(y)\langle y\rangle^{r} d y, \quad\langle y\rangle:=\sqrt{1+|y|^{2}} .
$$

The notations which will be used in this paper are as follows: the Minkowski metric is given by $\operatorname{diag}(-1,1,1,1)$, and the Robertson-Walker metric is

$$
d s^{2}=-d t^{2}+R^{2}(t)\left(\left(d x^{1}\right)^{2}+\left(d x^{2}\right)^{2}+\left(d x^{3}\right)^{2}\right),
$$

where the scale factor $R$ is a function of time $t$ satisfying

$$
\dot{R}(t) \geq 0 \text {. }
$$

For simplicity we assume

$$
R(0)=1 .
$$

Greek indices run from 0 to 3 , and Latin indices from 1 to $3 . p^{\alpha}$ denotes a four dimensional vector, while $p$ denotes a three dimensional (contravariant) vector. Similarly, $v_{\alpha}$ denotes a four dimensional vector, while $v$ denotes a three dimensional covariant vector. To be precise,

$$
p=\left(p^{1}, p^{2}, p^{3}\right), \quad v=\left(v_{1}, v_{2}, v_{3}\right),
$$

while

$$
p^{0}=\sqrt{1+R^{2}(t)|p|^{2}}, \quad v^{0}=-v_{0}=\sqrt{1+R^{-2}(t)|v|^{2}}
$$

Note that

$$
p^{0}=v^{0} .
$$


The Einstein summation convention is used as $p_{\alpha} p^{\alpha}=\sum_{\alpha=0}^{3} p_{\alpha} p^{\alpha}$, where the indices are lowered by $p_{\alpha}=g_{\alpha \beta} p^{\beta}$ for a metric $g_{\alpha \beta}$. In some places we use $p_{i} p^{i}=$ $\sum_{i=1}^{3} p_{i} p^{i}$. The usual inner product $\cdot$ will only be used for three-dimensional vectors:

$$
p \cdot q=\sum_{i=1}^{3} p^{i} q^{i}, \quad v \cdot u=\sum_{i=1}^{3} v_{i} u_{i}
$$

and $|\cdot|$ is such that $|p|^{2}=p \cdot p$ and $|v|^{2}=v \cdot v$ as usual.

\section{Existence Results in the CASES OF BOUNDED KeRnels}

In this part we briefly review the results of [18, 19, 20, 21, where the kernels of the collision operators are assumed to be bounded. For instance, the homogeneous Boltzmann equation in Minkowski space is written in [21] as

$$
\partial_{t} f=\frac{1}{p^{0}} \iint S\left(p, q, p^{\prime}, q^{\prime}\right)\left(f^{\prime} f_{*}^{\prime}-f f_{*}\right) d \omega \frac{d q}{q^{0}} .
$$

An unknown quantity $S$ is introduced to play a role of a kernel of the collision operator. The collision kernel $S$ is assumed to be bounded uniformly on pre- and post-collisional momenta with an additional symmetry assumption:

$$
\begin{gathered}
0 \leq S \leq C, \\
S\left(p, q, p^{\prime}, q^{\prime}\right)=S\left(p^{\prime}, q^{\prime}, p, q\right),
\end{gathered}
$$

where $C$ is a fixed constant. The solution obtained in 21] should be understood as a mild solution. By integrating the equation (2.1) with respect to the time variable from 0 to $t$, we obtain the following integral equation:

$$
f(t, p)=f_{0}(p)+\int_{0}^{t} \frac{1}{p^{0}} \iint S\left(p, q, p^{\prime}, q^{\prime}\right)\left(f^{\prime} f_{*}^{\prime}-f f_{*}\right) d \omega \frac{d q}{q^{0}} d s .
$$

By saying that $f$ is a solution to the Boltzmann equation (2.1), we mean $f$ satisfies the integral equation (2.4) in $L^{1}$-sense for each $t$. The main theorem can be stated as follows.

Theorem 2.1 (Noutchegueme and Tetsadjio, 21]). Suppose that the collision kernel $S$ satisfies (2.2) -(2.3) for a given positive constant $C$. Let $r \in\left(0, \frac{1}{56 \pi C}\right]$ and $f_{0} \in X_{r}$ be given for

$$
X_{r}:=L^{1}\left(\mathbb{R}^{3}\right) \cap\{f \geq 0, \text { a.e. }\} \cap\left\{\|f\|_{L^{1}} \leq r\right\} .
$$

Then, the Cauchy problem for the homogeneous Boltzmann equation (2.4) in Minkowski space has a unique global solution $f \in C\left([0, \infty) ; X_{r}\right)$ satisfying

$$
\sup _{t \in[0, \infty)}\|f(t)\|_{L^{1}} \leq\left\|f_{0}\right\|_{L^{1}}
$$

The above result is extended to the cases of curved spacetimes in [19, 20, and the initial condition is improved to any arbitrary large initial data (note that the initial condition in the above theorem depends on the constant $C$, which is the upper bound of the collision kernel). The equation considered in [20] can be written as

$$
\partial_{t} f-2 \frac{\dot{R}}{R} \sum_{i=1}^{3} p^{i} \partial_{p^{i}} f=\frac{1}{p^{0}} \iint A\left(t, p, q, p^{\prime}, q^{\prime}\right)\left(f^{\prime} f_{*}^{\prime}-f f_{*}\right) d \omega \frac{R^{3} d q}{q^{0}},
$$


where the scale factor $R=R(t)$ is assumed to be given such that it is differentiable and bounded from below, and the collision kernel $A$ is similarly assumed to be bounded with a symmetry assumption:

$$
\begin{gathered}
R(t) \geq C, \\
0 \leq A \leq C, \\
A\left(t, p, q, p^{\prime}, q^{\prime}\right)=A\left(t, p^{\prime}, q^{\prime}, p, q\right) .
\end{gathered}
$$

The solution of the equation (2.5) obtained in 20] should also be understood as a mild solution. We define a characteristic curve of (2.5) by

$$
\frac{d p}{d t}=-2 \frac{\dot{R}}{R} p \quad \text { with } \quad p(0)=y, \quad \text { hence } \quad p(t)=R^{-2}(t) y .
$$

Then, the equation (2.5) can be written as

$$
\frac{d}{d t} f(t, p(t))=(\text { collision term })
$$

and by integrating the above equation in a similar way, we obtain

$$
f(t, p(t))=f_{0}(y)+\int_{0}^{t}(\text { collision term }) d s .
$$

However, if we regard $f$ as a function of $t$ and $y$, where $y$ and $p=p(t)$ are related to each other by (2.9), i.e.

$$
\frac{d}{d t} f(t, y)=(\text { collision term })
$$

then we obtain a different mild form,

$$
f(t, y)=f_{0}(y)+\int_{0}^{t}(\text { collision term }) d s,
$$

and this was the argument of [20. To be precise, we express the above integral equation in terms of $y$ and $z:=R^{2}(s) q$, and obtain the following mild form of the Boltzmann equation in the Robertson-Walker spacetime:

$$
f(t, y)=f_{0}(y)+\int_{0}^{t} \frac{1}{y^{0}} \iint A\left(s, y, z, y^{\prime}, z^{\prime}\right)\left(f^{\prime} f_{*}^{\prime}-f f_{*}\right) d \omega \frac{d z}{R^{3}(s) z^{0}} d s .
$$

The solutions obtained in 20 are functions which satisfy the integral equation (2.10) in $L^{1}$-sense for each $t$. If we recall that in this paper the Boltzmann equation in the Robertson-Walker spacetime refers to (1.5) and that to derive this form of the equation we considered covariant variables $v$ instead of $p$, then we can see that considering the characteristic curve corresponds to considering the covariant variables and that the new variable $y$ of (2.10) corresponds to the covariant variable $v$ of (1.5). The main theorem of [20] can be stated as follows.

Theorem 2.2 (Noutchegueme and Takou, 20). Suppose that the collision kernel A satisfies (2.6) -(2.8), and let $f_{0} \in L^{1}\left(\mathbb{R}^{3}\right)$ with $f_{0} \geq 0$ be given. Then, the Cauchy problem for the relativistic Boltzmann equation in the Robertson-Walker spacetime (2.10) has a unique global solution $f \in C\left([0, \infty) ; L^{1}\left(\mathbb{R}^{3}\right)\right)$ with $f(t) \geq 0$ satisfying

$$
\sup _{t \in[0, \infty)}\|f(t)\|_{L^{1}} \leq\left\|f_{0}\right\|_{L^{1}}
$$


In the above result the scale factor $R(t)$ is only assumed to be bounded from below, hence Theorem 2.2 includes Theorem 2.1. A similar result has been obtained in [19] in a little more general spacetime, which is a Bianchi Type I spacetime, and finally the Einstein-Boltzmann system has been studied in 18 . The solution space used in [18] is a weighted $L^{1}$ space, which corresponds to $L_{1}^{1}\left(\mathbb{R}^{3}\right)$ in the notation of the present paper. If we combine the arguments and proofs of [18, 19, 20, the following theorem is obtained.

Proposition 2.1. Consider the Boltzmann equation (2.10) in the Robertson-Walker spacetime. Suppose that the collision kernel $A$ satisfies (2.6)-(2.8), and let $f_{0} \in$ $L_{1}^{1}\left(\mathbb{R}^{3}\right)$ with $f_{0} \geq 0$ be given. Then, the Boltzmann equation has a unique global solution $f \in C\left([0, \infty) ; L_{1}^{1}\left(\mathbb{R}^{3}\right)\right)$ with $f(t) \geq 0$ satisfying

$$
\sup _{t \in[0, \infty)}\|f(t)\|_{L_{1}^{1}} \leq\left\|f_{0}\right\|_{L_{1}^{1}}
$$

\section{Existence Results For the hard POTENTIAL CASES}

In this section the existence result of Proposition 2.1 will be extended to the hard potential case. Proposition 2.1 shows that the Boltzmann equation (2.10) has a solution in the weighted function space $L_{1}^{1}\left(\mathbb{R}^{3}\right)$ with the weight function $\langle y\rangle$. By assuming that the collision kernel $A$ is independent of $t$ and the scale factor satisfies $R(t) \equiv 1$, we obtain the existence result in $L_{1}^{1}\left(\mathbb{R}^{3}\right)$ for the equation (2.4). This will be extended to the hard potential case in Section 3.2 . The existence result for the Robertson-Walker case will be studied in Section 3.3

3.1. Preliminaries. In this section we collect several lemmas.

Lemma 3.1. The following inequalities hold in the Robertson-Walker spacetime:

$$
\begin{gathered}
g \leq \sqrt{s} \\
g \leq 2 \sqrt{p^{0} q^{0}}, \\
\sqrt{s} \leq 2 \sqrt{p^{0} q^{0}}, \\
g \leq R(t)|p-q| .
\end{gathered}
$$

Proof. Since $s=g^{2}+4$, we obtain the first inequality. The second inequality is given by the first and the third ones. For the third inequality we notice that

$$
\begin{aligned}
s & =2+2 p^{0} q^{0}-2 R^{2}(t)(p \cdot q) \\
& =2 p^{0} q^{0}+2 \sqrt{1-2 R^{2}(t)(p \cdot q)+R^{4}(t)(p \cdot q)^{2}} \\
& \leq 2 p^{0} q^{0}+2 \sqrt{1+R^{2}(t)\left(|p|^{2}+|q|^{2}\right)+R^{4}(t)|p|^{2}|q|^{2}} \\
& =4 p^{0} q^{0},
\end{aligned}
$$

and this proves the third inequality. The last inequality is also clear because

$$
g^{2}=-\left(p^{0}-q^{0}\right)^{2}+R^{2}(t)|p-q|^{2} \leq R^{2}(t)|p-q|^{2},
$$

and this completes the proof.

Lemma 3.2. For any $\omega \in \mathbb{S}^{2}$, we have $t_{\alpha} t^{\alpha} \geq R^{2}(t) s$ in the Robertson-Walker spacetime. 
Proof. In the Robertson-Walker spacetime we have

$$
t^{\alpha}=\left(n_{i} \omega^{i},-n_{0} \omega\right)=\left(R^{2}(t)(n \cdot \omega), n^{0} \omega\right),
$$

and the proof is a direct calculation:

$$
\begin{aligned}
t_{\alpha} t^{\alpha} & =-R^{4}(t)(n \cdot \omega)^{2}+R^{2}(t)\left(n^{0}\right)^{2} \geq-R^{4}(t)|n|^{2}+R^{2}(t)\left(n^{0}\right)^{2} \\
& =R^{2}(t)\left(\left(n^{0}\right)^{2}-R^{2}(t)|n|^{2}\right)=R^{2}(t) s .
\end{aligned}
$$

This completes the proof.

The following lemma is a well-known fact in the special relativistic case 13, and we show that the lemma holds in general relativistic cases also. The lemma is proved by the same argument as in [13, but we present it for the reader's convenience.

Lemma 3.3. Let $\left(p^{\prime \alpha}, q^{\alpha}\right)$ and $\left(p^{\alpha}, q^{\alpha}\right)$ be pre- and post-collisional momenta respectively, and consider the collision map $\left(p^{\alpha}, q^{\alpha}\right) \rightarrow\left(p^{\prime \alpha}, q^{\prime \alpha}\right)$. Then, the Jacobian is given by

$$
\frac{\partial\left(p^{\prime}, q^{\prime}\right)}{\partial(p, q)}=-\frac{p_{0}^{\prime} q_{0}^{\prime}}{p_{0} q_{0}}
$$

Proof. To prove the lemma, we use a different parametrization from (1.1).

$$
p^{\alpha}=p^{\alpha}+2 \frac{t_{\beta} q^{\beta}}{t_{\gamma} t^{\gamma}} t^{\alpha}, \quad q^{\alpha}=q^{\alpha}-2 \frac{t_{\beta} q^{\beta}}{t_{\gamma} t^{\gamma}} t^{\alpha},
$$

where $t^{\alpha}$ is the same as in (1.1). For convenience we write

$$
p^{\prime k}=p^{k}+A \omega^{k}, \quad q^{\prime k}=q^{k}-A \omega^{k}, \quad A=-2 \frac{t_{\beta} q^{\beta}}{t_{\gamma} t^{\gamma}} n_{0} .
$$

By the same calculations as in 13 we obtain

$$
\begin{aligned}
\frac{\partial\left(p^{\prime}, q^{\prime}\right)}{\partial(p, q)} & =\operatorname{det}\left(\begin{array}{cc}
\delta_{j}^{i}+\left(\partial_{p^{j}} A\right) \omega^{i} & \left(\partial_{q^{j}} A\right) \omega^{i} \\
-\left(\partial_{p^{j}} A\right) \omega^{i} & \delta_{j}^{i}-\left(\partial_{q^{j}} A\right) \omega^{i}
\end{array}\right) \\
& =\operatorname{det}\left(\delta_{j}^{i}+\left(\partial_{p^{j}} A-\partial_{q^{j}} A\right) \omega^{i}\right) \\
& =1+\left(\partial_{p^{i}} A-\partial_{q^{i}} A\right) \omega^{i} .
\end{aligned}
$$

We differentiate the conserved energy

$$
p^{\prime 0}+q^{\prime 0}=p^{0}+q^{0}
$$

with respect to $p^{j}$, and multiply $\omega^{j}$ to obtain

$$
\begin{aligned}
& \left(-\frac{p_{k}^{\prime}}{p_{0}^{\prime}} \frac{\partial p^{\prime k}}{\partial p^{j}}-\frac{q_{k}^{\prime}}{q_{0}^{\prime}} \frac{\partial q^{\prime k}}{\partial p^{j}}\right) \omega^{j}=-\frac{p_{j}}{p_{0}} \omega^{j} \\
& \Longleftrightarrow \quad\left(\frac{p_{j}}{p_{0}}-\frac{p_{j}^{\prime}}{p_{0}^{\prime}}\right) \omega^{j}=\left(\frac{p_{k}^{\prime}}{p_{0}^{\prime}}-\frac{q_{k}^{\prime}}{q_{0}^{\prime}}\right) \omega^{k}\left(\partial_{p^{j}} A\right) \omega^{j} .
\end{aligned}
$$

Similarly we obtain

$$
\left(\frac{q_{j}}{q_{0}}-\frac{q_{j}^{\prime}}{q_{0}^{\prime}}\right) \omega^{j}=\left(\frac{p_{k}^{\prime}}{p_{0}^{\prime}}-\frac{q_{k}^{\prime}}{q_{0}^{\prime}}\right) \omega^{k}\left(\partial_{q^{j}} A\right) \omega^{j} .
$$

Hence, (3.5) is given by

$$
\frac{\partial\left(p^{\prime}, q^{\prime}\right)}{\partial(p, q)}=\left(\frac{p_{k}^{\prime}}{p_{0}^{\prime}} \omega^{k}-\frac{q_{k}^{\prime}}{q_{0}^{\prime}} \omega^{k}\right)^{-1}\left(\frac{p_{j}}{p_{0}} \omega^{j}-\frac{q_{j}}{q_{0}} \omega^{j}\right) .
$$


Recall that $n^{\alpha}=p^{\alpha}+q^{\alpha}$ and $t^{\alpha}=\left(n_{j} \omega^{j},-n_{0} \omega\right)$ for $\omega \in \mathbb{S}^{2}$, and then the above quantities are written as follows:

$$
\frac{p_{j}}{p_{0}} \omega^{j}-\frac{q_{j}}{q_{0}} \omega^{j}=\frac{1}{p_{0} q_{0}}\left(q_{0} n_{j}-n_{0} q_{j}\right) \omega^{j}=\frac{1}{p_{0} q_{0}} q_{\alpha} t^{\alpha} .
$$

Similarly we obtain

$$
\begin{aligned}
\frac{p_{k}^{\prime}}{p_{0}^{\prime}} \omega^{k}-\frac{q_{k}^{\prime}}{q_{0}^{\prime}} \omega^{k} & =\frac{1}{p_{0}^{\prime} q_{0}^{\prime}}\left(q_{0}^{\prime} p_{k}^{\prime}+q_{0}^{\prime} q_{k}^{\prime}-q_{0}^{\prime} q_{k}^{\prime}-p_{0}^{\prime} q_{k}^{\prime}\right) \omega^{k} \\
& =\frac{1}{p_{0}^{\prime} q_{0}^{\prime}}\left(q_{0}^{\prime} n_{k}-n_{0} q_{k}^{\prime}\right) \omega^{k}=\frac{1}{p_{0}^{\prime} q_{0}^{\prime}} q_{\alpha}^{\prime} t^{\alpha}=-\frac{1}{p_{0}^{\prime} q_{0}^{\prime}} q_{\alpha} t^{\alpha},
\end{aligned}
$$

where we used the energy-momentum conservation and the following:

$$
t_{\alpha} q^{\prime \alpha}=t_{\alpha} q^{\alpha}-2 \frac{t_{\beta} q^{\beta}}{t_{\gamma} t^{\gamma}} t_{\alpha} t^{\alpha}=-t_{\alpha} q^{\alpha} .
$$

We plug (3.7) and (3.8) into (3.6), and this completes the proof.

Lemma 3.4. For the collision operator the following property holds in the RobertsonWalker spacetime: for any measurable function $k$ depending only on $g, s$, and $\omega$, we have

$$
\begin{aligned}
& \iiint \frac{k(g, s, \omega)}{p^{0} q^{0}}\left(f^{\prime} f_{*}^{\prime}-f f_{*}\right)\left(p^{0}\right)^{r} d \omega d q d p \\
& =\frac{1}{2} \iiint \frac{k(g, s, \omega)}{p^{0} q^{0}} f f_{*}\left(\left(p^{\prime 0}\right)^{r}+\left(q^{\prime 0}\right)^{r}-\left(p^{0}\right)^{r}-\left(q^{0}\right)^{r}\right) d \omega d q d p .
\end{aligned}
$$

Proof. We use Lemma 3.3 to make the change of variables between pre- and postcollisional momenta as follows:

$$
\frac{1}{p^{0} q^{0}} d p d q=\frac{1}{p^{\prime 0} q^{0}} d p^{\prime} d q^{\prime}
$$

and note that $g$ and $s$ are invariant quantities under the collision process and symmetric for $p$ and $q$. Hence, the gain term can be written as

$$
\begin{aligned}
\iiint \frac{k(g, s, \omega)}{p^{0} q^{0}} f^{\prime} f_{*}^{\prime}\left(p^{0}\right)^{r} d \omega d q d p & =\iiint \frac{k(g, s, \omega)}{p^{0} q^{\prime 0}} f^{\prime} f_{*}^{\prime}\left(p^{0}\right)^{r} d \omega d q^{\prime} d p^{\prime} \\
& =\iiint \frac{k(g, s, \omega)}{p^{0} q^{0}} f f_{*}\left(p^{\prime 0}\right)^{r} d \omega d q d p .
\end{aligned}
$$

By interchanging $p$ and $q$, it can also be rewritten as

$$
\iiint \frac{k(g, s, \omega)}{p^{0} q^{0}} f^{\prime} f_{*}^{\prime}\left(p^{0}\right)^{r} d \omega d q d p=\iiint \frac{k(g, s, \omega)}{p^{0} q^{0}} f f_{*}\left(q^{\prime 0}\right)^{r} d \omega d q d p .
$$

Hence, we obtain the following representation for the gain term:

$$
\iiint \frac{k(g, s, \omega)}{p^{0} q^{0}} f^{\prime} f_{*}^{\prime}\left(p^{0}\right)^{r} d \omega d q d p=\frac{1}{2} \iiint \frac{k(g, s, \omega)}{p^{0} q^{0}} f f_{*}\left(\left(p^{\prime 0}\right)^{r}+\left(q^{\prime 0}\right)^{r}\right) d \omega d q d p .
$$

After applying the same argument to the loss term, we obtain the desired result.

Lemma 3.5. Consider the collision process in the Robertson-Walker spacetime. Let $\left(p^{\prime \alpha}, q^{\alpha}\right)$ and $\left(p^{\alpha}, q^{\alpha}\right)$ be pre- and post-collisional momenta respectively. Consider the following quantity for $r>1$ :

$$
G=\left(p^{\prime 0}\right)^{r}+\left(q^{\prime 0}\right)^{r}-\left(p^{0}\right)^{r}-\left(q^{0}\right)^{r} .
$$


Then, $G$ satisfies

$$
G \leq C_{r}\left(\left(p^{0}\right)^{r-1} q^{0}+p^{0}\left(q^{0}\right)^{r-1}\right) .
$$

If $\omega$ is restricted to a subset $\left\{\omega \in \mathbb{S}^{2}:|n \cdot \omega| \leq \frac{1}{\sqrt{2}}|n|\right\}$, then $G$ satisfies

$$
G \leq C_{r}\left(\left(p^{0}\right)^{r-\frac{1}{2}}\left(q^{0}\right)^{\frac{1}{2}}+\left(p^{0}\right)^{\frac{1}{2}}\left(q^{0}\right)^{r-\frac{1}{2}}\right)-c_{r}\left(\left(p^{0}\right)^{r}+\left(q^{0}\right)^{r}\right),
$$

where $C_{r}$ and $c_{r}$ are two different positive constants depending on $r$.

Proof. Note that $p^{\prime 0}+q^{\prime 0}=p^{0}+q^{0}$ is a conserved quantity for each $p^{0}$ and $q^{0}$. Let $p^{\alpha}$ and $q^{\alpha}$ be given. Then, $G$ reduces to a function of $\omega$ and can be written as

$$
\begin{aligned}
G(\omega) & =\left(p^{0}\right)^{r}+\left(q^{0}\right)^{r}-\left(p^{0}\right)^{r}-\left(q^{0}\right)^{r} \\
& =\left(p^{\prime 0}\right)^{r}+\left(p^{0}+q^{0}-p^{\prime 0}\right)^{r}-\left(p^{0}\right)^{r}-\left(q^{0}\right)^{r} .
\end{aligned}
$$

Note that $G$ has minimum at $p^{\prime 0}=\left(p^{0}+q^{0}\right) / 2$, i.e., when $p^{\prime 0}=q^{\prime 0}$, and is monotonically increasing as $p^{\prime 0}$ tends to 0 or $p^{0}+q^{0}$. Hence, $G$ attains its maximum when $p^{\prime 0}-q^{\prime 0}$ is extremal. Without loss of generality we may assume $p^{\prime 0} \geq q^{\prime 0}$. From (1.1) we obtain

$$
p^{\prime 0}-q^{\prime 0}=g \frac{t^{0}}{\sqrt{t_{\alpha} t^{\alpha}}}=g \frac{R^{2}(n \cdot \omega)}{\sqrt{R^{2}\left(n^{0}\right)^{2}-R^{4}(n \cdot \omega)^{2}}} .
$$

This quantity attains its maximum when $\omega$ is parallel to $n$, which means that $p^{\prime 0}$ is largest and $q^{\prime 0}$ is smallest when $n \cdot \omega=|n|$, in particular $q^{\prime 0} \leq \min \left\{p^{0}, q^{0}\right\}$, which implies again $\left(q^{0}\right)^{r} \leq \min \left\{\left(p^{0}\right)^{r},\left(q^{0}\right)^{r}\right\}$. Consequently, $G$ attains its maximum when $n \cdot \omega=|n|$ and is estimated as

$$
\begin{aligned}
G(\omega) & \leq G(n /|n|) \leq\left.\left(p^{0}\right)^{r}\right|_{\omega=n /|n|}-\max \left\{\left(p^{0}\right)^{r},\left(q^{0}\right)^{r}\right\} \\
& =\left.\left(\frac{p^{0}+q^{0}}{2}+\frac{g}{2} \frac{R^{2}|n|}{\sqrt{t_{\alpha} t^{\alpha}}}\right)^{r}\right|_{\omega=n /|n|}-\max \left\{\left(p^{0}\right)^{r},\left(q^{0}\right)^{r}\right\} .
\end{aligned}
$$

By applying Lemma 3.1, Lemma 3.2, and the inequality

$$
(a+b)^{r} \leq a^{r}+b^{r}+C_{r}\left(a^{r-1} b+a b^{r-1}\right) \quad \text { for } \quad r>1,
$$

we obtain

$$
\begin{aligned}
G(\omega) & \leq\left(\frac{p^{0}+q^{0}}{2}+\frac{R|p+q|}{2}\right)^{r}-\max \left\{\left(p^{0}\right)^{r},\left(q^{0}\right)^{r}\right\} \\
& \leq\left(p^{0}+q^{0}\right)^{r}-\max \left\{\left(p^{0}\right)^{r},\left(q^{0}\right)^{r}\right\} \\
& \leq \min \left\{\left(p^{0}\right)^{r},\left(q^{0}\right)^{r}\right\}+C_{r}\left(\left(p^{0}\right)^{r-1} q^{0}+p^{0}\left(q^{0}\right)^{r-1}\right) \\
& \leq C_{r}\left(\left(p^{0}\right)^{r-1} q^{0}+p^{0}\left(q^{0}\right)^{r-1}\right),
\end{aligned}
$$

and this proves the first result. 
To prove the second result, we take the assumption $|n \cdot \omega| \leq \frac{1}{\sqrt{2}}|n|$ and suppose $p^{\prime 0} \geq q^{\prime 0}$. Then, $p^{\prime 0}$ is estimated as

$$
\begin{aligned}
p^{\prime 0} & \leq \frac{p^{0}+q^{0}}{2}+\frac{g}{2} \frac{R^{2}|n \cdot \omega|}{\sqrt{R^{2}\left(n^{0}\right)^{2}-R^{4}(n \cdot \omega)^{2}}} \\
& \leq \frac{p^{0}+q^{0}}{2}+\frac{g}{2 \sqrt{2}} \frac{R^{2}|p+q|}{\sqrt{R^{2}\left(p^{0}+q^{0}\right)^{2}-\frac{1}{2} R^{4}|p+q|^{2}}} \leq \frac{p^{0}+q^{0}}{2}+\frac{g}{2} \\
& \leq \frac{\left(\sqrt{p^{0}}+\sqrt{q^{0}}\right)^{2}}{2},
\end{aligned}
$$

where we used Lemma 3.1. Then, $G$ is estimated as

$$
\begin{aligned}
G & \leq 2\left(p^{0}\right)^{r}-\left(p^{0}\right)^{r}-\left(q^{0}\right)^{r} \\
& \leq \frac{\left(\sqrt{p^{0}}+\sqrt{q^{0}}\right)^{2 r}}{2^{r-1}}-\left(p^{0}\right)^{r}-\left(q^{0}\right)^{r} \\
& \leq \frac{\left(p^{0}\right)^{r}}{2^{r-1}}+\frac{\left(q^{0}\right)^{r}}{2^{r-1}}+C_{r}\left(\left(p^{0}\right)^{r-\frac{1}{2}}\left(q^{0}\right)^{\frac{1}{2}}+\left(p^{0}\right)^{\frac{1}{2}}\left(q^{0}\right)^{r-\frac{1}{2}}\right)-\left(p^{0}\right)^{r}-\left(q^{0}\right)^{r} \\
& \leq C_{r}\left(\left(p^{0}\right)^{r-\frac{1}{2}}\left(q^{0}\right)^{\frac{1}{2}}+\left(p^{0}\right)^{\frac{1}{2}}\left(q^{0}\right)^{r-\frac{1}{2}}\right)-c_{r}\left(\left(p^{0}\right)^{r}+\left(q^{0}\right)^{r}\right),
\end{aligned}
$$

where we used (3.12). The constants $C_{r}$ and $c_{r}$ are two different positive constants depending on $r$, and this completes the proof.

Remark 3.1. Those types of inequalities given in Lemma 3.5 are called the Povzner inequality, which was originally proved by Povzner in [23]. This inequality has been crucially used to prove existence theorems for the non-relativistic spatially homogeneous Boltzmann equation by Elmroth 11] and Mischler and Wennberg [17. The sharpest form of the Povzner inequality is given by Mischler and Wennberg, but Lemma 3.5 corresponds to a relativistic extension of Elmroth's result.

3.2. Hard potential case in Minkowski space. Comparing the two forms (1.2) and (2.1) for the Boltzmann equation, we can see that the collision kernel $S$ of (2.1) corresponds to $g \sqrt{s} \sigma(g, \theta)$ of (1.2). Hence, if the quantity $g \sqrt{s} \sigma(g, \theta)$ is suitably truncated, then the truncated equation has a global solution by Proposition 2.1 For simplicity the following notations will be used:

$$
v_{\phi, m}:=\frac{\min \{g \sqrt{s}, m\}}{p^{0} q^{0}}, \quad g_{m}:=\min \{g, m\}, \quad \sigma_{0, m}(\omega):=\min \left\{\sigma_{0}(\omega), m\right\} .
$$

For each integer $m$, let $f_{m}$ be a solution of the following truncated equation with initial data $f_{m}(0)=f_{0}$ :

$$
\partial_{t} f_{m}=Q_{m}\left(f_{m}, f_{m}\right)
$$

where $Q_{m}$ is defined as

$$
Q_{m}(h, h):=\iint v_{\phi, m}\left(g_{m}\right)^{a} \sigma_{0, m}(\omega)\left(h^{\prime} h_{*}^{\prime}-h h_{*}\right) d \omega d q .
$$

Then, the truncated equation has a unique global solution $f_{m} \in C\left([0, \infty) ; L_{1}^{1}\left(\mathbb{R}^{3}\right)\right)$ by Proposition 2.1. Consequently, a sequence $\left\{f_{m}\right\}$ is obtained, and existence of solutions for the original equation (1.2) is proved by showing that the sequence $\left\{f_{m}\right\}$ is a Cauchy sequence in $L_{1}^{1}\left(\mathbb{R}^{3}\right)$. This argument was used for the non-relativistic case by Mischler and Wennberg in [17, and below we will show that their argument is applicable to the relativistic case. 
Lemma 3.6. For any $r \geq 0$ and $T>0$, there exists a constant $C_{r}$ which does not depend on $m$ such that if $\left\|f_{0}\right\|_{1, r}$ is bounded, then

$$
\sup _{m} \sup _{t \in[0, T]}\left\|f_{m}(t)\right\|_{1, r} \leq C_{r} .
$$

Proof. We first note that by Proposition 2.1

$$
\sup _{t \in[0, \infty)}\left\|f_{m}(t)\right\|_{1, r} \leq C \quad \text { for } \quad 0 \leq r \leq 1,
$$

where $C$ does not depend on $m$, and for $r \leq s$,

$$
\left\|f_{m}(t)\right\|_{1, r} \leq\left\|f_{m}(t)\right\|_{1, s}
$$

We now assume $r>1$. By direct calculations we have

$$
\begin{aligned}
& \frac{d}{d t}\left\|f_{m}(t)\right\|_{1, r} \\
& =\iiint v_{\phi, m}\left(g_{m}\right)^{a} \sigma_{0, m}(\omega)\left(f_{m}^{\prime} f_{m *}^{\prime}-f_{m} f_{m *}\right)\left(p^{0}\right)^{r} d \omega d q d p \\
& =\frac{1}{2} \iiint v_{\phi, m}\left(g_{m}\right)^{a} \sigma_{0, m}(\omega) f_{m} f_{m *}\left(\left(p^{0}\right)^{r}+\left(q^{0}\right)^{r}-\left(p^{0}\right)^{r}-\left(q^{0}\right)^{r}\right) d \omega d q d p
\end{aligned}
$$

where we used Lemma 3.4. We apply Lemma 3.5 to obtain

$$
\frac{d}{d t}\left\|f_{m}(t)\right\|_{1, r} \leq I_{1}+I_{2}-I_{3}
$$

where

$$
\begin{aligned}
& I_{1}=C_{r} \iiint_{|n \cdot \omega| \geq \frac{1}{\sqrt{2}}|n|} v_{\phi, m}\left(g_{m}\right)^{a} \sigma_{0, m}(\omega) f_{m} f_{m *}\left(\left(p^{0}\right)^{r-1} q^{0}+p^{0}\left(q^{0}\right)^{r-1}\right) d \omega d q d p, \\
& I_{2}=C_{r} \iiint_{|n \cdot \omega| \leq \frac{1}{\sqrt{2}}|n|} v_{\phi, m}\left(g_{m}\right)^{a} \sigma_{0, m}(\omega) f_{m} f_{m *}\left(\left(p^{0}\right)^{r-\frac{1}{2}}\left(q^{0}\right)^{\frac{1}{2}}+\left(p^{0}\right)^{\frac{1}{2}}\left(q^{0}\right)^{r-\frac{1}{2}}\right) d \omega d q d p, \\
& I_{3}=c_{r} \iiint_{|n \cdot \omega| \leq \frac{1}{\sqrt{2}}|n|} v_{\phi, m}\left(g_{m}\right)^{a} \sigma_{0, m}(\omega) f_{m} f_{m *}\left(\left(p^{0}\right)^{r}+\left(q^{0}\right)^{r}\right) d \omega d q d p .
\end{aligned}
$$

The second term $I_{2}$ is easily estimated by using Lemma 3.1 as

$$
I_{2} \leq C_{r} \iint f_{m} f_{m *}\left(p^{0}\right)^{r-\frac{1}{2}+\frac{a}{2}}\left(q^{0}\right)^{\frac{1}{2}+\frac{a}{2}} d q d p \leq C_{r}\left\|f_{m}(t)\right\|_{1, r-\frac{1}{2}+\frac{a}{2}}\left\|f_{m}(t)\right\|_{1, \frac{1}{2}+\frac{a}{2}} .
$$

Consider now $\sigma_{0, m}(\omega)$, which is defined by

$$
\sigma_{0, m}(\omega):=\min \left\{\sin ^{\gamma} \theta, m\right\} \quad \text { for } \quad-2<\gamma \leq-1 .
$$

Note that $\sigma_{0, m}(\omega)$ is integrable on $\mathbb{S}^{2}$ for $\gamma>-2$, and there exists a constant $C_{\gamma}$ satisfying $\int_{\mathbb{S}^{2}} \sigma_{0, m}(\omega) d \omega \leq C_{\gamma}$, where the constant $C_{\gamma}$ does not depend on $m$. On the other hand, since $\gamma$ is negative, we have $\sigma_{0, m}(\omega) \geq 1$ for any $m$. Moreover, the integration domain of $I_{3}$ is a set with Lebesgue measure

$$
\mu\{|n \cdot \omega| \leq|n| / \sqrt{2}\}=2 \sqrt{2} \pi,
$$

which does not depend on $m$. Hence, $I_{1}$ and $I_{3}$ can be estimated as

$$
\begin{aligned}
& I_{1} \leq D_{r} \iint_{\mathbb{R}^{6}} v_{\phi, m}\left(g_{m}\right)^{a} f_{m} f_{m *}\left(p^{0}\right)^{r-1} q^{0} d q d p, \\
& I_{3} \geq d_{r} \iint_{\mathbb{R}^{6}} v_{\phi, m}\left(g_{m}\right)^{a} f_{m} f_{m *}\left(q^{0}\right)^{r} d q d p,
\end{aligned}
$$


HO LEE AND ALAN D. RENDALL

for some constants $D_{r}$ and $d_{r}$. We now fix the constants $D_{r}$ and $d_{r}$ to split the domain by $\left\{D_{r}\left(p^{0}\right)^{r-1} \leq d_{r}\left(q^{0}\right)^{r-1}\right\}$ and $\left\{D_{r}\left(p^{0}\right)^{r-1} \geq d_{r}\left(q^{0}\right)^{r-1}\right\}$, and then obtain $I_{1} \leq I_{11}+I_{12}$, where

$$
\begin{aligned}
& I_{11}=D_{r} \iint_{D_{r}\left(p^{0}\right)^{r-1} \leq d_{r}\left(q^{0}\right)^{r-1}} v_{\phi, m}\left(g_{m}\right)^{a} f_{m} f_{m *}\left(p^{0}\right)^{r-1} q^{0} d q d p, \\
& I_{12}=D_{r} \iint_{D_{r}\left(p^{0}\right)^{r-1} \geq d_{r}\left(q^{0}\right)^{r-1}} v_{\phi, m}\left(g_{m}\right)^{a} f_{m} f_{m *}\left(p^{0}\right)^{r-1} q^{0} d q d p .
\end{aligned}
$$

We now obtain

$$
I_{11} \leq I_{3}
$$

In the case of $I_{12}$, we may simply use $\left(g_{m}\right)^{a} \leq C\left(p^{0} q^{0}\right)^{\frac{a}{2}} \leq C_{r}\left(p^{0}\right)^{a}$. Then, $I_{12}$ is easily estimated as

$$
I_{12} \leq C_{r}\left\|f_{m}(t)\right\|_{1, r-1+a}
$$

Combining the above estimates, we obtain

$$
\begin{aligned}
& \frac{d}{d t}\left\|f_{m}(t)\right\|_{1, r} \\
& \leq C_{r}\left(\left\|f_{m}(t)\right\|_{1, r-\frac{1}{2}+\frac{a}{2}}\left\|f_{m}(t)\right\|_{1, \frac{1}{2}+\frac{a}{2}}+\left\|f_{m}(t)\right\|_{1, r-1+a}\right) \leq C_{r}\left\|f_{m}(t)\right\|_{1, r},
\end{aligned}
$$

where we used the fact that $0 \leq a \leq \gamma+2$ and $-2<\gamma \leq-1$. Then, the lemma is proved by applying Grönwall's inequality.

Lemma 3.7. Consider the sequence $\left\{f_{m}\right\}$ on any finite time interval $[0, T]$. For any small number $\delta>0$, there exists a positive integer $M$ such that if $k, m \geq M$, then

$$
\sup _{t \in[0, T]}\left\|f_{k}(t)-f_{m}(t)\right\|_{1,1} \leq \delta .
$$

Proof. Let $k \leq m$ be two positive integers. By direct calculations,

$$
\frac{d}{d t}\left\|f_{k}(t)-f_{m}(t)\right\|_{1,1}=I+J
$$

where

$$
\begin{aligned}
& I=\int \operatorname{sgn}\left(f_{k}-f_{m}\right)\left(Q_{k}\left(f_{k}, f_{k}\right)-Q_{k}\left(f_{m}, f_{m}\right)\right) p^{0} d p, \\
& J=\int \operatorname{sgn}\left(f_{k}-f_{m}\right)\left(Q_{k}\left(f_{m}, f_{m}\right)-Q_{m}\left(f_{m}, f_{m}\right)\right) p^{0} d p,
\end{aligned}
$$

and $I$ and $J$ will be estimated separately. The first term $I$ is split again as

$$
\begin{aligned}
I= & \frac{1}{2} \iiint \operatorname{sgn}\left(f_{k}-f_{m}\right) v_{\phi, k}\left(g_{k}\right)^{a} \sigma_{0, k}(\omega) \\
& \times\left(\left(f_{k}^{\prime}-f_{m}^{\prime}\right)\left(f_{k *}^{\prime}+f_{m *}^{\prime}\right)+\left(f_{k}^{\prime}+f_{m}^{\prime}\right)\left(f_{k *}^{\prime}-f_{m *}^{\prime}\right)\right. \\
& \left.\quad-\left(f_{k}-f_{m}\right)\left(f_{k *}+f_{m *}\right)-\left(f_{k}+f_{m}\right)\left(f_{k *}-f_{m *}\right)\right) p^{0} d \omega d q d p \\
= & I_{1}+I_{2}+I_{3}+I_{4} .
\end{aligned}
$$

Each $I_{i}$ is estimated as follows:

$$
\begin{aligned}
I_{1} & \leq \frac{1}{2} \iiint v_{\phi, k}\left(g_{k}\right)^{a} \sigma_{0, k}(\omega)\left|f_{k}^{\prime}-f_{m}^{\prime}\right|\left(f_{k *}^{\prime}+f_{m *}^{\prime}\right) p^{0} d \omega d q d p \\
& =\frac{1}{2} \iiint v_{\phi, k}\left(g_{k}\right)^{a} \sigma_{0, k}(\omega)\left|f_{k}-f_{m}\right|\left(f_{k *}+f_{m *}\right) p^{\prime 0} d \omega d q d p
\end{aligned}
$$




$$
\begin{gathered}
I_{2} \leq \frac{1}{2} \iiint v_{\phi, k}\left(g_{k}\right)^{a} \sigma_{0, k}(\omega)\left(f_{k}^{\prime}+f_{m}^{\prime}\right)\left|f_{k *}^{\prime}-f_{m *}^{\prime}\right| p^{0} d \omega d q d p \\
=\frac{1}{2} \iiint v_{\phi, k}\left(g_{k}\right)^{a} \sigma_{0, k}(\omega)\left(f_{k *}+f_{m *}\right)\left|f_{k}-f_{m}\right| q^{\prime 0} d \omega d q d p \\
I_{3}=-\frac{1}{2} \iiint \operatorname{sgn}\left(f_{k}-f_{m}\right) v_{\phi, k}\left(g_{k}\right)^{a} \sigma_{0, k}(\omega)\left(f_{k}-f_{m}\right)\left(f_{k *}+f_{m *}\right) p^{0} d \omega d q d p \\
=-\frac{1}{2} \iiint v_{\phi, k}\left(g_{k}\right)^{a} \sigma_{0, k}(\omega)\left|f_{k}-f_{m}\right|\left(f_{k *}+f_{m *}\right) p^{0} d \omega d q d p
\end{gathered}
$$

and finally

$$
\begin{aligned}
I_{4} & \leq \frac{1}{2} \iiint v_{\phi, k}\left(g_{k}\right)^{a} \sigma_{0, k}(\omega)\left(f_{k}+f_{m}\right)\left|f_{k *}-f_{m *}\right| p^{0} d \omega d q d p \\
& \leq \frac{1}{2} \iiint v_{\phi, k}\left(g_{k}\right)^{a} \sigma_{0, k}(\omega)\left(f_{k *}+f_{m *}\right)\left|f_{k}-f_{m}\right| q^{0} d \omega d q d p .
\end{aligned}
$$

Therefore, $I$ is estimated as

$$
\begin{aligned}
I & \leq \frac{1}{2} \iiint v_{\phi, k}\left(g_{k}\right)^{a} \sigma_{0, k}(\omega)\left|f_{k}-f_{m}\right|\left(f_{k *}+f_{m *}\right)\left(p^{\prime 0}+q^{\prime 0}-p^{0}+q^{0}\right) d \omega d q d p \\
& \leq C \iint\left(g_{k}\right)^{a}\left|f_{k}-f_{m}\right|\left(f_{k *}+f_{m *}\right) q^{0} d q d p,
\end{aligned}
$$

where we used

$$
p^{\prime 0}+q^{\prime 0}=p^{0}+q^{0} .
$$

By using $g_{k} \leq 2 \sqrt{p^{0} q^{0}}$, we obtain for $I$

$$
\begin{aligned}
I & \leq C \iint\left|f_{k}-f_{m}\right|\left(f_{k *}+f_{m *}\right)\left(p^{0}\right)^{\frac{a}{2}}\left(q^{0}\right)^{1+\frac{a}{2}} d q d p \\
& \leq C \sup _{n}\left\|f_{n}(t)\right\|_{1,1+\frac{a}{2}}\left\|f_{k}(t)-f_{m}(t)\right\|_{1, \frac{a}{2}} .
\end{aligned}
$$

To estimate the second term $J$, we note that

$$
\begin{aligned}
\left|v_{\phi, k}-v_{\phi, m}\right| & =\frac{1}{p^{0} q^{0}}|\min \{g \sqrt{s}, k\}-\min \{g \sqrt{s}, m\}| \\
& \leq \mathbf{1}_{\{g \sqrt{s} \geq k\}} \frac{\min \{g \sqrt{s}, m\}}{p^{0} q^{0}}=\mathbf{1}_{\{g \sqrt{s} \geq k\}} v_{\phi, m},
\end{aligned}
$$

and similarly

$$
\begin{aligned}
& \left|\left(g_{k}\right)^{a}-\left(g_{m}\right)^{a}\right| \leq \mathbf{1}_{\{g \geq k\}}\left(g_{m}\right)^{a}, \\
& \left|\sigma_{0, k}(\omega)-\sigma_{0, m}(\omega)\right| \leq \mathbf{1}_{\left\{\sin ^{\gamma} \theta \geq k\right\}} \sigma_{0, m}(\omega) .
\end{aligned}
$$

Hence, $J$ can be estimated as

$$
\begin{aligned}
J \leq & \iiint\left|v_{\phi, k}\left(g_{k}\right)^{a} \sigma_{0, k}(\omega)-v_{\phi, m}\left(g_{m}\right)^{a} \sigma_{0, m}(\omega)\right|\left|f_{m}^{\prime} f_{m *}^{\prime}-f_{m} f_{m *}\right| p^{0} d \omega d q d p \\
\leq & \iiint \mathbf{1}_{\{g \sqrt{s} \geq k\}} v_{\phi, m}\left(g_{k}\right)^{a} \sigma_{0, k}(\omega)\left(f_{m}^{\prime} f_{m *}^{\prime}+f_{m} f_{m *}\right) p^{0} d \omega d q d p \\
& +\iiint \mathbf{1}_{\{g \geq k\}} v_{\phi, m}\left(g_{m}\right)^{a} \sigma_{0, k}(\omega)\left(f_{m}^{\prime} f_{m *}^{\prime}+f_{m} f_{m *}\right) p^{0} d \omega d q d p \\
& +\iiint \mathbf{1}_{\{\sin \gamma \theta \geq k\}} v_{\phi, m}\left(g_{m}\right)^{a} \sigma_{0, m}(\omega)\left(f_{m}^{\prime} f_{m *}^{\prime}+f_{m} f_{m *}\right) p^{0} d \omega d q d p \\
= & : J_{1}+J_{2}+J_{3} .
\end{aligned}
$$


Note that each $J_{i}$ can be separated into two terms: a gain term containing $f_{m}^{\prime} f_{m *}^{\prime}$ and a loss term containing $f_{m} f_{m *}$. The gain and loss terms are estimated in the same way after making the change of variables $(p, q) \leftrightarrow\left(p^{\prime}, q^{\prime}\right)$, hence we only present the estimates for the loss terms. To estimate $J_{1}$, we take a small number $\varepsilon>0$ and use $g \sqrt{s} \leq 4 p^{0} q^{0}$ from Lemma 3.1

$$
\begin{aligned}
J_{1} & \leq C \iint \mathbf{1}_{\left\{4 p^{0} q^{0} \geq k\right\}}\left(g_{k}\right)^{a} f_{m} f_{m *} p^{0} d q d p \\
& \leq C \iint \mathbf{1}_{\left\{4 p^{0} q^{0} \geq k\right\}} f_{m}\left(p^{0}\right)^{1+\frac{a}{2}} f_{m *}\left(q^{0}\right)^{\frac{a}{2}} d q d p \\
& \leq \frac{C}{k^{\varepsilon}} \iint \mathbf{1}_{\left\{4 p^{0} q^{0} \geq k\right\}} f_{m}\left(p^{0}\right)^{1+\frac{a}{2}+\varepsilon} f_{m *}\left(q^{0}\right)^{\frac{a}{2}+\varepsilon} d q d p \\
& \leq \frac{C}{k^{\varepsilon}}\left\|f_{m}(t)\right\|_{1,1+\frac{a}{2}+\varepsilon}\left\|f_{m}(t)\right\|_{1, \frac{a}{2}+\varepsilon} .
\end{aligned}
$$

To estimate $J_{2}$, we use $g \leq|p-q|$ to obtain

$$
\begin{aligned}
J_{2} & \leq C \iint \mathbf{1}_{\{|p-q| \geq k\}} f_{m}\left(p^{0}\right)^{1+\frac{a}{2}} f_{m *}\left(q^{0}\right)^{\frac{a}{2}} d q d p \\
& \leq C \iint \mathbf{1}_{\left\{|p| \geq \frac{k}{2}\right\} \cup\left\{|q| \geq \frac{k}{2}\right\}} f_{m}\left(p^{0}\right)^{1+\frac{a}{2}} f_{m *}\left(q^{0}\right)^{\frac{a}{2}} d q d p \\
& \leq C\left\|f_{m}(t)\right\|_{1,1+\frac{a}{2}} \int \mathbf{1}_{\left\{|q| \geq \frac{k}{2}\right\}} f_{m *}\left(q^{0}\right)^{\frac{a}{2}} d q \\
& \leq \frac{C}{k}\left\|f_{m}(t)\right\|_{1,1+\frac{a}{2}}^{2} .
\end{aligned}
$$

For $J_{3}$ term, we use $\sin \theta \approx \theta$ for $0 \leq \theta \leq \frac{\pi}{2}$. Hence, the condition $\sin ^{\gamma} \theta \geq k$ is equivalent to $\theta \leq C k^{\frac{1}{\gamma}}$ since $\gamma$ is negative. We first estimate $J_{3}$ as

$$
\begin{aligned}
J_{3} & \left.\leq C \iiint \mathbf{1}_{\{\theta \leq C k} \frac{1}{\gamma}\right\} \\
& \leq C\left\|f_{m}(t)\right\|_{1,1+\frac{a}{2}}\left\|f_{m}(t)\right\|_{1, \frac{a}{2}} \int \mathbf{1}_{\left\{\theta \leq C k^{\frac{1}{\gamma}}\right\}} \sigma_{0, m}(\omega) d \omega .
\end{aligned}
$$

The integration on $\mathbb{S}^{2}$ above is estimated as

$$
\int \mathbf{1}_{\left\{\theta \leq C k^{\frac{1}{\gamma}}\right\}} \sigma_{0, m}(\omega) d \omega \leq 2 \pi \int_{0}^{C k^{\frac{1}{\gamma}}} \sin ^{\gamma+1} \theta d \theta \leq C k^{\frac{\gamma+2}{\gamma}},
$$

where the constant depends on $\gamma$. Note that $-1 \leq(\gamma+2) / \gamma<0$, and the third term $J_{3}$ is estimated as

$$
J_{3} \leq C k^{\frac{\gamma+2}{\gamma}}\left\|f_{m}(t)\right\|_{1,1+\frac{a}{2}}\left\|f_{m}(t)\right\|_{1, \frac{a}{2}} .
$$

We combine (3.14), (3.15), (3.16), and (3.17), and apply Lemma 3.6 on any finite time interval $[0, T]$ to obtain

$$
\begin{aligned}
\frac{d}{d t}\left\|f_{k}(t)-f_{m}(t)\right\|_{1,1} & \leq C\left(k^{-\varepsilon}+k^{-1}+k^{\frac{\gamma+2}{\gamma}}\right)+C\left\|f_{k}(t)-f_{m}(t)\right\|_{1, \frac{a}{2}} \\
& \leq C\left(k^{-\varepsilon}+k^{\frac{\gamma+2}{\gamma}}\right)+C\left\|f_{k}(t)-f_{m}(t)\right\|_{1,1} .
\end{aligned}
$$

Since $f_{k}(0)=f_{m}(0)$ and $(\gamma+2) / \gamma$ is negative, we obtain the desired result by applying Grönwall's inequality.

We now obtain the following theorem. 
Theorem 3.1. Suppose that the scattering kernel has the form (1.6) and initial data satisfies $f_{0} \in L_{r}^{1}\left(\mathbb{R}^{3}\right)$ for $r>1+\frac{a}{2}$ with $f_{0} \geq 0$. Then, the Boltzmann equation (1.2) in Minkowski space has a unique global solution $f \in C\left([0, \infty) ; L_{1}^{1}\left(\mathbb{R}^{3}\right)\right)$ with $f(t) \geq 0$.

Proof. Lemma 3.7 shows that the sequence $\left\{f_{m}\right\}$ is a Cauchy sequence in $L_{1}^{1}\left(\mathbb{R}^{3}\right)$. Hence, there exists a solution $f$ to the Boltzmann equation (1.2). The initial condition $f_{0} \in L_{r}^{1}\left(\mathbb{R}^{3}\right)$ with $r>1+\frac{a}{2}$ comes from (3.15), and nonnegativity of the solution is guaranteed by Proposition 2.1. Uniqueness of solutions can be easily proved by following the calculations given in the proof of Lemma 3.7. The initial condition of the theorem is rather strong in the sense that $L_{1}^{1}$ solutions are obtained from $L_{r}^{1}$ initial data for $r>1+\frac{a}{2}$, hence the proof of uniqueness is much easier than that of [17. This completes the proof of the theorem.

3.3. Hard potential case in the Robertson-Walker spacetime. In this part we extend the existence result of Theorem 3.1 to the Robertson-Walker case. The argument is basically the same as in the Minkowski case. We first truncate a certain part of the collision kernel, then existence for the truncated equation is guaranteed by Proposition 2.1. We obtain a sequence of solutions to the truncated equations, and by showing that the sequence is a Cauchy sequence we obtain a solution.

To deal with the Boltzmann equation (1.5) in the Robertson-Walker spacetime, we use the covariant variable $v$. In the Minkowski case we have $p^{0}=\langle p\rangle$, so there is no difference between the following quantities:

$$
\int f(t, p)\left(p^{0}\right)^{r} d p=\int f(t, p)\langle p\rangle^{r} d p .
$$

However, in the Robertson-Walker case we have

$$
v^{0}=\sqrt{1+R^{-2}(t)|v|^{2}} \text { and }\langle v\rangle=\sqrt{1+|v|^{2}} .
$$

If we introduce a new quantity:

$$
|f(t)|_{1, r}:=\int f(t, v)\left(v^{0}\right)^{r} d v,
$$

then we have the following relation:

$$
|f(t)|_{1, r} \leq\|f(t)\|_{1, r} \leq R^{r}(t)|f(t)|_{1, r},
$$

where we used

$$
v^{0} \leq\langle v\rangle \leq R(t) v^{0}
$$

Note that

$$
|f(0)|_{1, r}=\|f(0)\|_{1, r},
$$

since we assume $R(0)=1$.

The main goal of this section is to extend Theorem 3.1 to the Robertson-Walker case. We will show that the lemmas in the previous section can be applied to the Robertson-Walker case. To do this we first use $v^{0}$ as a weight function, i.e. we estimate $|f(t)|_{1, r}$, because all the calculations in the Minkowski case are naturally extended to the Robertson-Walker case when using $v^{0}$ instead of $\langle v\rangle$, for instance we can use the energy-momentum conservation. Then, by the relation (3.18) we obtain 
estimates for $\|f(t)\|_{1, r}$. Similarly to the Minkowski case we modify the Boltzmann equation (1.5) as

$$
\partial_{t} f_{m}=Q_{m}\left(f_{m}, f_{m}\right):=R^{-3} \iint v_{\phi, m}\left(g_{m}\right)^{a} \sigma_{0, m}(\omega)\left(f_{m}^{\prime} f_{m *}^{\prime}-f_{m} f_{m *}\right) d \omega d u,
$$

where

$$
v_{\phi, m}=\frac{\min \{g \sqrt{s}, m\}}{v^{0} u^{0}}, \quad g_{m}=\min \{g, m\}, \quad \sigma_{0, m}=\min \left\{\sigma_{0}(\omega), m\right\} .
$$

Then, the truncated equation has a unique global solution by Proposition 2.1. The following lemmas show that Lemma 3.6 and 3.7 can be extended to the RobertsonWalker case.

Lemma 3.8. For any $r \geq 0$ and $T>0$, there exists a constant $C_{r}$ which does not depend on $m$ such that if $\left\|f_{0}\right\|_{1, r}$ is bounded, then

$$
\sup _{m} \sup _{t \in[0, T]}\left|f_{m}(t)\right|_{1, r}+\left\|f_{m}(t)\right\|_{1, r} \leq C_{r} .
$$

Proof. We first estimate $\left|f_{m}(t)\right|_{1, r}$, and then obtain the desired result by using the relation (3.18). By Proposition 2.1 and the relation (3.18) we have

$$
\sup _{t \in[0, \infty)}\left|f_{m}(t)\right|_{1, r} \leq C \quad \text { for } \quad 0 \leq r \leq 1
$$

and now assume $r>1$. In the Robertson-Walker case, $v^{0}$ depends on time and decreases as time evolves for each $v$. To be precise,

$$
v^{0}=\sqrt{1+R^{-2}(t)|v|^{2}} \text { and } \quad \partial_{t} v^{0}=-\frac{\dot{R}(t)}{R^{3}(t)} \frac{|v|^{2}}{v^{0}} \leq 0,
$$

since we assume $R(t) \geq 1$ and $\dot{R}(t) \geq 0$. If we follow the calculation of the proof of Lemma 3.6. then we obtain

$$
\begin{aligned}
& \frac{d}{d t}\left|f_{m}(t)\right|_{1, r} \\
& =\frac{R^{-3}(t)}{2} \iiint v_{\phi, m}\left(g_{m}\right)^{a} \sigma_{0, m}(\omega) f_{m} f_{m *}\left(\left(v^{\prime 0}\right)^{r}+\left(u^{\prime 0}\right)^{r}-\left(v^{0}\right)^{r}-\left(u^{0}\right)^{r}\right) d \omega d u d v \\
& \quad+\int f_{m}(t, v) \frac{\partial}{\partial t}\left[\left(v^{0}\right)^{r}\right] d v,
\end{aligned}
$$

and the second integral is negative. Hence, we may only consider

$$
\begin{aligned}
& \frac{d}{d t}\left|f_{m}(t)\right|_{1, r} \\
& \leq \frac{R^{-3}(t)}{2} \iiint v_{\phi, m}\left(g_{m}\right)^{a} \sigma_{0, m}(\omega) f_{m} f_{m *}\left(\left(v^{\prime 0}\right)^{r}+\left(u^{\prime 0}\right)^{r}-\left(v^{0}\right)^{r}-\left(u^{0}\right)^{r}\right) d \omega d u d v,
\end{aligned}
$$

and follow the same calculations of Lemma 3.6 to obtain

$$
\sup _{m} \sup _{t \in[0, T]}\left|f_{m}(t)\right|_{1, r} \leq C_{r} .
$$

Consequently, the relation (3.18) gives the desired result. 
Lemma 3.9. Consider the sequence $\left\{f_{m}\right\}$ on any finite time interval $[0, T]$. For any small number $\delta>0$, there exists a positive number $M$ such that if $k, m \geq M$, then

$$
\sup _{t \in[0, T]}\left\|f_{k}(t)-f_{m}(t)\right\|_{1,1} \leq \delta .
$$

Proof. Similarly to the previous lemma, we first estimate $\left|f_{k}-f_{m}\right|_{1,1}$ and then obtain the desired result by using (3.18). By direct calculation, we have

$$
\begin{aligned}
& \frac{d}{d t}\left|f_{k}(t)-f_{m}(t)\right|_{1,1}=\frac{d}{d t} \int\left|f_{k}(t, v)-f_{m}(t, v)\right| v^{0} d v \\
& =\int \partial_{t}\left[\left|f_{k}(t, v)-f_{m}(t, v)\right|\right] v^{0}+\left|f_{k}(t, v)-f_{m}(t, v)\right| \partial_{t} v^{0} d v \\
& =\int \operatorname{sgn}\left(f_{k}-f_{m}\right)\left(Q_{k}\left(f_{k}, f_{k}\right)-Q_{m}\left(f_{m}, f_{m}\right)\right) v^{0} d v \\
& \quad-\frac{\dot{R}(t)}{R^{3}(t)} \int\left|f_{k}(t, v)-f_{m}(t, v)\right| \frac{|v|^{2}}{v^{0}} d v \\
& \leq \int \operatorname{sgn}\left(f_{k}-f_{m}\right)\left(Q_{k}\left(f_{k}, f_{k}\right)-Q_{m}\left(f_{m}, f_{m}\right)\right) v^{0} d v .
\end{aligned}
$$

Hence, we can follow the proof of Lemma 3.7 and obtain a positive number $M$ such that if $k, m \geq M$, then

$$
\sup _{t \in[0, T]}\left|f_{k}(t)-f_{m}(t)\right|_{1,1} \leq \delta .
$$

Consequently, we obtain the desired result by applying (3.18), and this completes the proof.

By the same argument as in Theorem 3.1, we obtain the following theorem.

Theorem 3.2. Suppose that the scattering kernel has the form of (1.6) and initial data satisfies $f_{0} \in L_{r}^{1}\left(\mathbb{R}^{3}\right)$ for $r>1+\frac{a}{2}$ with $f_{0} \geq 0$. Then, the Boltzmann equation (1.5) in the Robertson-Walker spacetime has a unique global solution $f \in$ $C\left([0, \infty) ; L_{1}^{1}\left(\mathbb{R}^{3}\right)\right)$ with $f(t) \geq 0$.

\section{Summary and outlook}

In this paper global existence theorems have been proved for spatially homogeneous solutions of the Boltzmann equation in Minkowski space and in spatially flat Robertson-Walker spacetimes. This was done for a class of collision kernels of hard potential type and these theorems extend existing results for smooth collision kernels with compact support to cases which are closer to those which naturally arise in physical problems.

There are many directions in which this work might be generalized. Do the special relativistic solutions constructed here converge to equilibrium as $t \rightarrow \infty$ ? If so, can a useful analogue be proved for the Robertson-Walker spacetimes? Note that in the latter case equilibrium is impossible since the existence of an equilibrium solution of the Boltzmann equation in a spacetime implies the existence of a timelike Killing vector field ([15, p. 1167) and vector fields of this kind do not exist in most Robertson-Walker spacetimes. It is nevertheless the case that in practise ideas related to equilibrium matter distributions are used in cosmology and so it should be possible to formulate some mathematical analogue of equilibrium solutions in an expanding cosmological model. 
Now that a global existence result has been obtained in a class of homogeneous and isotropic cosmological models it is natural to ask whether a similar result can be proved in general homogeneous cosmological models which expand for ever. In this paper the device of writing the equation in terms of the covariant components of the momentum is used to simplify the equation. In fact this trivializes the kinetic part of the equation. The same trick would work in more general models of Bianchi type I but not in general homogeneous models. It is nevertheless the case that this transformation does produce some simplification in general and this has been exploited in the study of the late-time behaviour of the Einstein-Vlasov system in 22 .

Another generalization is to look at the global existence question for homogeneous solutions of the Einstein-Boltzmann system. A template for this could be provided by the results of this type for the Einstein equations coupled to other matter models proved in [24]. For the class of collision kernels considered here there are no local existence theorems available for inhomogeneous solutions of the Einstein-Boltzmann system. There are global existence theorems available for the Boltzmann equation with this type of kernel in special relativity with small or close to homogeneous initial data. The small data problem for the Einstein-Boltzmann system is out of reach at present since even the corresponding problem for the Einstein-Vlasov system has not been solved. On the other hand in the presence of a positive cosmological constant there are global existence results for solutions of the Einstein-Vlasov system evolving from data which are close to homogeneous [26].

It is well-known that the Boltzmann equation is not well-posed in the past time direction. In cosmology this equation is of interest for the very early universe and so it is natural to enquire if solutions of the Einstein-Boltzmann system can be constructed which extend all the way back to the big bang. It is not reasonable to construct these by evolving backwards in time and a possible alternative is to pose data at the singularity. A formal study of this type of procedure has been carried out in 30 but corresponding existence proofs have not yet been developed. In conclusion, the study of the Boltzmann equation in curved spacetimes and its coupling to the Einstein equations gives rise to a variety of challenging mathematical problems.

\section{REFERENCES}

[1] Andréasson, H.: The Einstein-Vlasov system/kinetic theory. Living Rev. Relativity 14 (2011), 4, http://www.livingreviews.org/lrr-2011-4

[2] Arkeryd, L.: On the Boltzmann equation. Arch. Rational Mech. Anal. 45 (1972), 1-34.

[3] Bancel, D.: Problème de Cauchy pour l'équation de Boltzmann en relativité générale. Ann. Inst. H. Poincaré Sect. A (N.S.) 18 (1973), 263-284.

[4] Bancel, D., Choquet-Bruhat, Y.: Existence, uniqueness, and local stability for the EinsteinMaxwell-Boltzmann system. Commun. Math. Phys. 33 (1973), 83-96.

[5] Carleman, T.: Sur la théorie de l'équation intégrodifférentielle de Boltzmann. Acta Math. 60 (1933), no. 1, 91-146.

[6] Cercignani, C., Illner, R., Pulvirenti, M.: The mathematical theory of dilute gases. Applied Mathematical Sciences, 106. Springer-Verlag, New York, 1994.

[7] Choquet-Bruhat, Y.: General relativity and the Einstein equations. Oxford Mathematical Monographs. Oxford University Press, Oxford, 2009.

[8] de Groot, S. R., van Leeuwen, W. A., van Weert, C. G.: Relativistic kinetic theory. Principles and applications. North-Holland Publishing Co., Amsterdam-New York, 1980. 
[9] Dudyński, M., Ekiel-Jeżewska, M.: On the linearized relativistic Boltzmann equation. I. Existence of solutions. Comm. Math. Phys. 115 (1988), no. 4, 607-629.

[10] Ehlers, J.: General relativity and kinetic theory. General relativity and cosmology (Proc. Internat. School of Physics "Enrico Fermi", Italian Phys. Soc., Varenna, 1969), pp. 1-70. Academic Press, New York, 1971.

[11] Elmroth, T.: Global boundedness of moments of solutions of the Boltzmann equation for forces of infinite range. Arch. Rational Mech. Anal. 82 (1983), no. 1, 1-12.

[12] Glassey, R. T.: The Cauchy problem in kinetic theory. SIAM, Philadelphia, PA, 1996.

[13] Glassey, R. T., Strauss, W. A.: On the derivatives of the collision map of relativistic particles. Transport Theory Statist. Phys. 20 (1991), no. 1, 55-68.

[14] Glassey, R. T., Strauss, W. A.: Asymptotic stability of the relativistic Maxwellian. Publ. Res. Inst. Math. Sci. 29 (1993), no. 2, 301-347.

[15] Israel, W.: Relativistic kinetic theory of a simple gas. J. Math. Phys. 4 (1963), 1163-1181.

[16] Lee, H., Rendall, A. D.: The Einstein-Boltzmann system and positivity. To appear in J. Hyperbolic Differ. Equ.

[17] Mischler, S., Wennberg, B.: On the spatially homogeneous Boltzmann equation. Ann. Inst. H. Poincaré Anal. Non Linéaire 16 (1999), no. 4, 467-501.

[18] Noutchegueme, N. Dongo, D.: Global existence of solutions for the Einstein-Boltzmann system in a Bianchi type I spacetime for arbitrary large initial data. Classical Quantum Gravity 23 (2006), no. 9, 2979-3003.

[19] Noutchegueme, N., Dongo, D., Takou, E.: Global existence of solutions for the relativistic Boltzmann equation with arbitrary large initial data on a Bianchi Type I space-time. Gen. Relativity Gravitation 37 (2005), no. 12, 2047-2062.

[20] Noutchegueme, N., Takou, E.: Global existence of solutions for the relativistic Boltzmann equation on the flat Robertson-Walker space-time for arbitrary large initial data. Preprint arXiv:gr-qc/0507035 2 .

[21] Noutchegueme, N., Tetsadjio, M. E.: Global solutions for the relativistic Boltzmann equation in the homogeneous case on the Minkowski space-time. Preprint arXiv:gr-qc/0307065 1.

[22] Nungesser, E.: Future non-linear stability for solutions of the Einstein-Vlasov system of Bianchi types II and $\mathrm{VI}_{0}$. Preprint arXiv:1208.6158

[23] Povzner, A. J.: On the Boltzmann equation in the kinetic theory of gases. Mat. Sb. (N.S.) 58 (1962), 65-86.

[24] Rendall, A. D.: Global properties of locally spatially homogeneous cosmological models with matter. Math. Proc. Camb. Phil. Soc. 118 (1995), 511-526.

[25] Rendall, A. D.: Partial differential equations in general relativity. Oxford Graduate Texts in Mathematics, 16. Oxford University Press, Oxford, 2008.

[26] Ringström, H. Future stability of models of the universe - with an introduction to the EinsteinVlasov system. Book to appear with Oxford University Press.

[27] Stewart, J. M.: Non-equilibrium relativistic kinetic theory. Lecture Notes in Physics, 10. Springer-Verlag, Berlin-Heidelberg-New York, 1971.

[28] Strain, R. M.: Asymptotic stability of the relativistic Boltzmann equation for the soft potentials. Comm. Math. Phys. 300 (2010), no. 2, 529-597.

[29] Strain, R. M.: Coordinates in the relativistic Boltzmann theory. Kinet. Relat. Models 4 (2011), no. 1, 345-359.

[30] Tod. K. P.: Isotropic cosmological singularities: other matter models. Class. Quantum Grav. 20 (2003), 521-534.

[31] Wald, R. M.: General relativity. University of Chicago Press, Chicago, IL, 1984. 